\title{
Ein blinder Fleck der Diversitätsdebatte? Zur Neuguineaisierung der Stadt
}

\author{
Peter Dirksmeier \\ Leibniz Universität Hannover, Institut für Wirtschafts- und Kulturgeographie, Scheiderberg 50, 30167 \\ Hannover, Germany \\ Correspondence: Peter Dirksmeier (dirksmeier@kusogeo.uni-hannover.de)
}

Received: 15 November 2019 - Revised: 30 April 2020 - Accepted: 16 June 2020 - Published: 13 July 2020

\begin{abstract}
Kurzfassung. The contemporary metropolis is characterised by an increasing diversity of its population, which is called "super-diversity" or "hyperdiversity" in the urban discourse. This epistemological realignment breaks new ground away from the classic Chicago School. Associated with this "diversification of diversity" (Vertovec, 2007:1025) is a fragmentation of the different social and ethnic groups in the city. However, the concept of super-diversity camouflages a separateness of these groups that is called newguineaisation in this paper. With that said, this new urban reality claims for empirical investigations of the societal implication of this phenomenon especially with regard to social cohesion in contemporary cities.
\end{abstract}

\section{Neuguineaisierung der Stadt?}

Neuguinea stellt eine aus kultur- und sozialwissenschaftlicher Perspektive besondere Region dar. Keine Kolonialmacht vermochte es, in den ersten 400 Jahren der Kolonialgeschichte nachhaltig auf der Insel Fuß zu fassen und sie zu unterwerfen (Biskup et al., 1968). Die derzeit mindestens 750 verschiedenen bekannten Papua-Sprachen bilden ein Kaleidoskop von größter sprachlicher Verschiedenheit bei gleichzeitiger hoher struktureller Komplexität der jeweiligen Sprachen auf geringer Fläche. Dies führt dazu, dass jede Sprache zugleich eine ethnische Gruppe konstituiert. Die resultierenden ethnischen Gruppen nehmen sich jeweils wieder wechselseitig als Fremde wahr (Wurm, 1982). Der Versuch, eine ethnologisch-taxonomische Ordnung der Vielschichtigkeit und Diversität ethnischer Gruppen in Neuguinea zu (er)finden, eine Forschungspraxis, die Assmann (1996:27) in Anlehnung an Erik H. Erikson als ,,pseudo-speciation“ kritisierte, scheiterte zunächst an dem Problem kultureller Grenzziehungen (Schlesier, 1970).

Die ethnisch-kulturelle Komplexität bildet sich ebenfalls in der Stadtgesellschaft von Port Moresby ab, der Hauptstadt des auf der östlichen Inselhälfte gelegenen Staates PapuaNeuguinea. Urbanität stellt sich in Port Moresby als ein Universum aus Kinship-Kontakten dar. Organisationen mit Mitgliedschaftsregelungen, wie z.B. Kirchen, Arbeitgeben- de oder Sportvereine, rekurrieren auf ethnische Zugehörigkeit, auf Kosten ihrer potenziellen Integrationsfähigkeit für die (Stadt)Gesellschaft (Connell und Lea, 1995). Letztlich ist der Staat Papua-Neuguinea aus einer westlichen Perspektive (Anderson, 2016) eine ,nation of minorities“ (Knight, 1982:523) von sich wechselseitig als fremd beobachtenden Gruppen ohne übergreifende Integrationsformel.

In Analogie zu der soziokulturellen Situation in PapuaNeuguinea lässt sich von den gegenwartsgesellschaftlichen Großstädten im Globalen Norden als cities of minorities sprechen (Schneider et al., 2015), da die im Wesentlichen auf dem demografischen Wandel und Migrationsprozessen aufruhende, zunehmende Diversität der Stadtbevölkerung ebenfalls das tradierte Mehrheits-/Minderheitsverhältnis von Autochthonen und Fremden sprengt. Schneider et al. (2015:19) prognostizieren beispielsweise für die Großstädte in Deutschland eine Veränderung hin zu einer ,,mehrheitlich Minderheiten-Stadt“, deren Kennzeichen ist, dass es keine ethnisch definierte Mehrheitsbevölkerung mehr geben wird. In der ,,mehrheitlich Minderheiten-Stadt“ des Globalen Nordens werden die verschiedenen ethnischen Gruppen, die intern wiederum sozial diversifiziert sind, sich wechselseitig als Minderheiten unter Minderheiten begegnen (Schneider et al., 2015:19). Die Sozialwissenschaft hat auf diesen empirischen Trend bereits begrifflich reagiert. Das Konzept der 
Diversität, verstanden als die Wahrscheinlichkeit, dass zwei zufällig aus einer Population gezogene Individuen derselben Gruppe angehören (Simpson, 1949), wurde zu einer „SuperDiversität“ (Vertovec, 2007) bzw. „Hyperdiversität“" (Amin, 2012) angepasst. Super-Diversität beschreibt eine ,diversification of diversity“ (Vertovec, 2007:1025) und sei als Begriff insofern berechtigt, da insbesondere die Großstädte in Westeuropa zunehmend von einem dynamischen Wechselverhältnis zwischen einer steigenden Anzahl an neuen, verstreuten, von vielerlei Herkunft seienden, transnational vernetzten, sozioökonomisch stark differenzierten und stratifizierten Zuwanderinnen und Zuwanderern geprägt würden (Vertovec, 2007).

Der Diskurs um Super-Diversität in der Sozialgeographie hat sich seit der Publikation von Steven Vertovecs Paper im Jahr 2007 in kurzer Zeit bereits deutlich ausdifferenziert und fragt auf der einen Seite nach der der Rolle von SuperDiversität an den Ausgangspunkten von Migrationsbewegungen für das Leben in super-diversen Ankunftsquartieren in Metropolen des Globalen Nordens (Simic, 2019). Neben dieser erfolgten räumlichen Weitung auf die Quellgebiete von Migrationsbewegungen zeichnet sich eine weitere Ausdifferenzierung des Super-Diversitätsbegriffs ab, die „HyperDiversität" als Begriff für eine Form der theoretischen Analyse von Differenzierungen vorschlägt, die neben ethnischen, demographischen und sozioökonomischen Kategorien der Super-Diversität zusätzlich noch Haltungen, Einstellungen, Lebensstile und Materialitäten in den Blick nimmt (Kraftl et al., 2019:1191). Hyper-Diversität beschreibt folglich die $D i$ versifizierung von Super-Diversität im Globalen Norden, die insbesondere in Begegnungen von Bedeutung sei (Peterson, 2017; Butcher, 2019).

Empirische Arbeiten zeigen jedoch auf, dass diese theoretisch hergeleitete hyper-diversifizierte Super-Diversität nicht zwingend Interaktion und Emergenz bedeuten muss (Blokland und van Eijk, 2010). Die Literatur skizziert vielmehr eine Plethora an Diversitäten mit der Tendenz zur Zersplitterung und Überlagerung von Gruppenzuschreibungen, die in immer weitergehender Differenzierung zu zerrinnen scheinen. Diese Arbeiten verlieren zusehends die Frage aus dem Blick, wie Gesellschaft unter den genannten Bedingungen möglich ist. Das Argument dieses Kurzbeitrags ist vielmehr, dass sich häufig in den Großstädten im Globalen Norden eine soziokulturelle Situation einstellt, für die ich den Begriff der Neuguineaisierung vorschlage. Die Neuguineaisierung der Stadt kennzeichnet, dass auf der einen Seite viele Menschen gerade die soziokulturelle (Super-)Diversität in der Stadt suchen, diese damit gleichzeitig verstärken und in super-diverse Viertel ziehen, um beispielsweise an dem kulturell ausdifferenzierten Dienstleistungsangebot zu partizipieren. Auf der anderen Seite ist empirisch häufig festzustellen, dass soziale Interaktion zwischen den diversen ethnischen und sozialen Gruppen nur in geringem Maße ausgeprägt ist. Der Wunsch nach dem Erleben von Diversität wird weitestgehend ohne Erleben von gruppenübergreifender sozialer Interaktion realisiert (Blokland und van Eijk, 2010; Neal und Vincent, 2013).

\section{Probleme des Diversitätsdiskurses}

Der Begriff der Neuguineaisierung der Stadt beschreibt ein Phänomen in der „,mehrheitlich Minderheiten-Stadt“ (Schneider et al., 2015:19) und deckt gleichzeitig einen blinden Fleck des gegenwärtigen (Super-)Diversitätsdiskurses auf. Super-Diversität ist ursprünglich als Reaktion auf die Chicago School der Stadtsoziologie konzipiert. Steven Vertovec kritisiert mit seinem Begriff die aus seiner Perspektive im Jahr 2007 gegebene Situation, dass der überwiegende Teil der sozialwissenschaftlichen Studien zur Immigration und Stadt nach wie vor auf Paradigmen der Chicago School aufruhe (Vertovec, 2007). Gruppen würden zunächst ethnisch definiert und anschließend in Hinblick auf den Grad ihrer Akkulturation bzw. Assimilation hin untersucht. Dies bemesse sich beispielsweise nach Veränderung des sozioökonomischen Status, Segregation, sprachlichem Wandel oder interethnischen Ehen (Vertovec, 2007). Steven Vertovec kritisiert gerade die vorgenommenen Simplifizierungen der Migrationsforschung, die er an Begriffen wie Adaption, Assimilation, Integration, Inkorporation oder Insertion festmacht (Ralph und Staeheli, 2011). Damit bezieht sich die Kritik letztlich auf die Figuration von Mehrheit/Minderheit, die allen diesen Konzepten zugrunde liegt. Steven Vertovec kritisiert einen basalen Dualismus der Chicago School, der Differenz letztlich nur auf der Individualebene zulässt, auf der Makroebene hingegen lediglich einen vollständigen „Normenkonsens" kenne (Löw, 2001:121).

Schaut man in die Arbeiten der Chicago School, dann wird diese verschiedentlich kritisierte dualistische Struktur des Vokabulars mehr als deutlich. Robert Park erkannte, dass eine Organisation der Gesellschaft, wie fragil diese auch sein mochte, nur möglich ist, wenn es zu einer permanenten Neuorganisation des instabilen Verhältnisses von Individuen und sozialen Gruppen kommt, die untereinander in Konkurrenz stehen (Park, 1915). Als ein Intermediär in Robert Parks Denken erscheint hier der ,,marginal man“ als Individuum, das in zwei unterschiedlichen kulturellen Gruppen lebt (Park, 1928). Allerdings interessiert Robert Park am ,,marginal man“ nicht primär die dieser Denkfigur eingeschriebene grundlegende Möglichkeit einer Dissolution der Binarität von Individuum und Struktur, sondern vielmehr die Taktiken und Wege zur Erreichung von sozialen Rollen in der Mehrheitsgesellschaft, die der „marginal man“ anwendet. Wesentlich ist hier das Konzept des Vorurteils, das die mögliche Rollenerreichung gerade inhibiert. Damit ist die wichtige Erweiterung des Diversitätsdiskurses durch den Begriff der Hyper-Diversität auf Einstellungen (Kraftl et al., 2019:1191) bereits in Chicago angelegt. Robert Park nennt beispielhaft als meist unerreichbare soziale Rollen für den „marginal man“ die Figur des Nachbarn oder des Bürgers 
(Park, 1950). Betrachtet man die wichtigsten empirischen „Lebensgeschichten“ (Damisch, 1997:39), die die Chicago School tatsächlich geschrieben hat, dann zeigt sich, dass die anzutreffenden Taktiken und Strategien (de Certeau, 1988) der Immigrantinnen und Immigranten in ihrer Auseinandersetzung mit der Mehrheitsgesellschaft und damit in einer Figuration der hierarchischen Komplementarität von Mehrheit/Minderheit, wobei die Mehrheit die Minderheit repräsentiert und definiert (Dirksmeier, 2016), zu verstehen, das ureigene Anliegen der Stadtsoziologie Chicagoer Provenienz ist.

Die in den Arbeiten der Chicago School maßgebliche Figuration der hierarchischen Komplementarität von Mehrheit/Minderheit ist empirisch nicht mehr haltbar. Vielmehr reagiert der jüngere Diversitätsdiskurs mit Begriffen wie Super-Diversität und Hyper-Diversität auf die neue, sich abzeichnende Situation einer ,,mehrheitlich MinderheitenStadt" (Schneider et al., 2015:19), indem er Differenzen in Bezug auf Aufenthaltstitel und nachfolgende soziale Auswirkungen - wie Arbeitslosigkeit, Illegalität, divergierende Arbeitsmarkterfahrungen, unterschiedliche Konfigurationen von Gender und Alter, neue Muster räumlicher Verteilung und gemischte Reaktionen der lokalen Wohnkontexte durch Bewohnerinnen und Bewohner und örtliche Dienstleistende, aber auch Einstellungen und Wertehaltung der Minderheiten - in Rechnung stellt (Kraftl et al., 2019; Vertovec, 2019). Diese neuen Diversitätskonzepte bezeichnen ebenfalls die Interaktion der beteiligten Attribute. Im Ergebnis produzieren diese Interaktionen neue hierarchisch strukturierte Statuspositionen und Stratifizierungen, die wiederum zu neuen Mustern sozialer Ungleichheit und Vorurteilen wie Rassismus, Segregation, Kosmopolitismus oder Kreolisierung führen (Vertovec, 2019).

Mein Argument ist, dass die begriffliche Erfassung der Konstellation von sich wechselseitig als Minderheiten unter Minderheiten begegnenden Gruppen in der Summe einen Großteil der Forschungsaufmerksamkeit auf sich zieht. Im Ergebnis entsteht eine Fülle an Diversitätskonzepten, die zwar die empirische Auflösung der hierarchischen Komplementarität von Mehrheit/Minderheit in den westlichen Großstädten bis hin zu Einstellungsdimensionen unter den beteiligten Gruppen beschreibt, aber die Tendenz zur fehlenden Interaktion und Emergenz zwischen den unterschiedlichen Gruppen, was ich mit Neuguineaisierung der Stadt bezeichne, eher verdeckt. Der Begriff der Super-Diversität beispielsweise, verstanden als ein Abrücken von den Dualismen der Chicago School, wirkt darauf hin, dass nicht länger die Frage von Minorität vs. Majorität oder die Konzeption von ethnischen Gruppen den entscheidenden Maßstab der Diversitätsforschung darstellt, sondern, dass diese sich auf kategoriale Differenzen umstellt, die Interaktionen zwischen Gruppen tangieren oder die Ergebnisse von Zurechnungsleistungen modifizieren (Piekut et al., 2012). Gleichzeitig laufen empirische Forschungen zur Super-Diversität Gefahr, das Narrativ der hierarchischen Komplementarität von Mehrheit/Minderheit zu reproduzieren. Solche Studien zielen dann auf Konflikte zwischen meist zwei Parteien und bilden letztlich die Stadtsoziologie aus Chicago ab. Vertovec (2019:128) selbst kritisiert die empirische Umsetzung der Super-Diversität als beliebig: ,scholars invoke superdiversity as a new condition or setting, and then carry on with describing whatever set of research findings they wish to present". Diese Beliebigkeit überdeckt die forschungslogische Notwendigkeit, neben der begrifflichen Erfassung der gegenwartsgesellschaftlichen Diversität, den gesellschaftlich hoch relevanten Aspekt der wechselseitigen Abgrenzung und Zersplitterung in den Blick zu nehmen. Soziale Kohäsion als eine basale Bedingung eines funktionierenden Gemeinwesens, inkl. positiver Haltungen sowie aufeinander bezogener Praktiken und Institutionen, erscheint dann als gefährdet, wenn Städte aufgrund ihrer diversen Struktur nicht länger als „Integrationsmaschine“ für unterschiedlichste Gruppen fungieren können (Krämer-Badoni, 2001:13). Die Aufgabe stellt sich folglich, für die Analyse der wesentlichen gesellschaftlichen Prozesse der Diversitätsfiguration im 21. Jahrhundert eine ähnlich tragfähige theoretische Basis zu finden, wie es der Chicago School für das 20. Jahrhundert und der dominierenden Figuration von Mehrheit/Minderheit gelang.

Die vorgeschlagene Semantik der Neuguineaisierung erlaubt es in diesem Zusammenhang, vier unterschiedliche und bis dato begrifflich getrennte Entwicklungen in den Großstädten des Globalen Nordens zusammen zu fassen und forschungslogisch zu zentrieren:

a. Die tradierte Konstellation von Mehrheit/Minderheit löst sich in eine Situation auf, in der Minderheiten nebeneinander leben (Schneider et al., 2015). Etablierte Begriffe wie Parallelgesellschaft, Ghetto(isierung), Enklave oder Diaspora werden mit ihrer - in der Herkunft dieser Begriffe aus der Chicagoer Schule der Stadtsoziologie begründeten - Fokussierung auf die Mehrheit/Minderheit-Konstellation dieser Lebenswirklichkeit nicht länger gerecht.

b. Gleichzeitig besteht eine soziale Distanzierung der Minderheiten untereinander, wie sie Emory Bogardus bereits zu Anfang des letzten Jahrhunderts mit dem Begriff der sozialen Distanz beschrieben hat (Bogardus, 1925). Unerwünschte, zufällige Kontakte werden durch Umzüge vermieden (Stillwell, 2010) und zugleich wird ein Leben als Mehrheit in einem Stadtteil präferiert, unabhängig von möglicherweise bestehenden Vorurteilen gegenüber anderen ethnischen Gruppen (Dirksmeier, 2014; Ehrkamp, 2008). Zusätzlich weisen empirische Arbeiten zur Segregation von hochqualifizierten Migrantinnen und Migranten nach, dass diese oft die am stärksten segregierte Gruppe in einer Stadt stellen, etwa Japanerinnen und Japaner in Düsseldorf (White, 1998). Super-Diversität (oder Hyper-Diversität) geht folglich häufig ohne sozialen Austausch und nachfolgend Hybridität (Bhabha, 2004) einher. 
c. Dies führt zu einer deutlichen sozialen Fragmentierung auf Basis von Unsicherheiten dieser neuen Situation gegenüber (Heeg, 2014), die wiederum Fragen nach der Möglichkeit von sozialer Ordnung und sozialem $\mathrm{Zu}$ sammenhalt (Schiefer und van der Noll, 2017) aufwirft. Diese Fragmentierung ist geeignet, an Grundfesten des sozialen Zusammenhalts zu rütteln, wie die Akzeptanz von Redistribution (Brady und Finnigan, 2014).

d. Der Begriff verdeutlicht zuletzt die Notwendigkeit für die Entwicklung von Forschungsperspektiven zur Erfassung von lokalen praktischen Umgangsweisen mit den spezifischen soziokulturellen Situationen in den cities of minorities, wie sie etwa im Urbanitätsdiskurs der Sozialgeographie bereits eingefordert werden (Haferburg und Rothfuss, 2019).

Super-Diversität oder Hyper-Diversität bedeutet nicht einfach ein Mehr an Pluralität als ein Nebeneinanderbestehen von Verschiedenem, sondern sie können mit einer Neuguineaisierung einhergehen als bewusstes, aktives voneinander Abgrenzen ethnisch definierter Gruppen in den cities of minorities im Globalen Norden, die keine Mehrheitsbevölkerung mehr kennen (Schneider et al., 2015). Damit entsteht eine Situation der Gleichzeitigkeit von ethnischer Segregation und hochgradiger ethnischer Diversität (Holloway et al., 2012), die bis dato noch wenig reflektiert wird. SuperDiversität und Hyper-Diversität greifen als Begriffe (noch) zu kurz, da die Konzepte, aufruhend auf der Annahme von Migration als Quelle von Diversität (Piekut et al., 2012), den Ist-Zustand der gegenwärtigen Pluralität als „Diversifizierung der Diversität“ (Vertovec, 2007) bzw. Diversifizierung der Super-Diversität widergeben, aber die soziale Ausgestaltung gesellschaftlichen Lebens in den neuguineaisierten $\mathrm{ci}$ ties of minorities bis dato zu wenig konzeptualisieren.

Datenverfügbarkeit. Für diesen Artikel wurden keine Datensätze genutzt.

Interessenkonflikt. Der Autor erklärt, dass kein Interessenkonflikt besteht.

Danksagung. Ich danke Nadine Marquardt und zwei anonymen Gutachter*innen für ihre wertvollen und hilfreichen Kritiken und Anregungen.

Begutachtung. This paper was edited by Nadine Marquardt and reviewed by two anonymous referees.

\section{Literatur}

Amin, A.: Land of Strangers, Polity Press, Cambridge, Malden, 2012.

Anderson, B.: Imagined Communities. Reflections on the Origin and Spread of Nationalism, Verso, London, New York, 2016.

Assmann, J.: Translating gods: religion as a factor of cultural (un)translatability, in: The Translatability of Cultures. Figurations of the Space Between, edited by: Budick, S. and Iser, W., Stanford University Press, Stanford, 25-36, 1996.

Bhabha, H. K.: The Location of Culture, Routledge, London, New York, 2004.

Biskup, P., Jinks, B., and Nelson, H.: A Short History of New Guinea, Angus \& Robertson, London, 1968.

Blokland, T. and van Eijk, G.: Do people who like diversity practice diversity in neighbourhood life? Neighbourhood use and the social networks of "diversity-seekers" in a mixed neighbourhood in the Netherlands, J. Ethn. Migr. Stud., 36, 313-332, https://doi.org/10.1080/13691830903387436, 2010.

Bogardus, E.: Social distance and its origin, J. Appl. Sociol., 9, 216226, 1925.

Brady, D. and Finnigan, R.: Does immigration undermine public support for social policy?, Am. Sociol. Rev., 79, 17-42, https://doi.org/10.1177/0003122413513022, 2014.

Butcher, M.: Re-working encounter: the role of reflexivity in managing difference, Soc. Cult. Geogr., 20, 1198-1217, https://doi.org/10.1080/14649365.2017.1346198, 2019.

Connell, J. and Lea, J.: Distant places, other cities? Urban life in contemporary Papua New Guinea, in: Postmodern Cities and Spaces, edited by: Gibson, K. and Watson, S., Blackwell, Cambridge, Oxford, 165-183, 1995.

Damisch, H.: Skyline. Architektur als Denkform, Passagen Verlag, Wien, 1997.

de Certeau, M.: Kunst des Handelns, Merve Verlag, Berlin, 1988.

Dirksmeier, P.: Are urbanites more permissive? Germany's urban geography of prejudice, Urban Aff. Rev., 50, 835-863, https://doi.org/10.1177/1078087414520950, 2014.

Dirksmeier, P.: Providing places for structures of feeling and hierarchical complementarity in urban theory: Re-reading Williams' The Country and the City, Urban Stud., 53, 884-898, https://doi.org/10.1177/0042098015571241, 2016.

Ehrkamp, P.: Risking publicity: masculinities and the racialization of public neighborhood space, Soc. Cult. Geogr., 9, 117-133, https://doi.org/10.1080/14649360701856060, 2008.

Haferburg, C. and Rothfuss, E.: Relational urbanity. Perspectives of a global urban society beyond universalism and localism, Geogr. Z., 107, 166-187, https://doi.org/10.25162/GZ-2019-0011, 2019.

Heeg, S.: Fragmentierung, in: Schlüsselbegriffe der Kultur- und Sozialgeographie, Herausgeber: Lossau, J., Freytag, T., und Lippuner, R., Ulmer, Stuttgart, 67-80, 2014.

Holloway, S. R., Wright, R., and Ellis, M.: The racially fragmented city? Neighborhood racial segregation and diversity jointly considered, Prof. Geogr., 64, 63-82, https://doi.org/10.1080/00330124.2011.585080, 2012.

Knight, D. B.: Identity and territory: geographical perspectives on nationalism and regionalism, Ann. Assoc. Am. Geogr., 72, 514 531, https://doi.org/10.1111/j.1467-8306.1982.tb01842.x, 1982. 
Kraftl, P., Bolt, G., and Van Kempen, R.: Hyper-diversity in/and geographies of childhood and youth, Soc. Cult. Geogr., 20, 1189-1197, https://doi.org/10.1080/14649365.2018.1491619, 2019.

Krämer-Badoni, T.: Urbanität und gesellschaftliche Integration, Deutsche Zeitschrift für Kommunalwissenschaften, 40, 12-26, 2001.

Löw, M.: Gemeindestudien heute: Sozialforschung in der Tradition der Chicagoer Schule?, Zeitschrift für qualitative Bildungs-, Beratungs- und Sozialforschung, 2, 111-131, 2001.

Neal, S. and Vincent, C.: Multiculture, middle class competencies and friendship practices in super-diverse geographies, Soc. Cult. Geogr., 14, 909-929, https://doi.org/10.1080/14649365.2013.837191, 2013.

Park, R. E.: The city: suggestions for the investigation of human behavior in the city environment, Am. J. Sociol., 20, 577-612, https://doi.org/10.1086/212433, 1915.

Park, R. E.: Human migration and the marginal man, Am J. Sociol., 33, 881-893, https://doi.org/10.1086/214592, 1928.

Park, R. E.: Personality and cultural conflict, in: Race and Culture, edited by: Park, R. E., The Free Press, Glencoe, 357-371, 1950.

Peterson, M.: Living with difference in hyper-diverse areas: How important are encounters in semi-public spaces?, Soc. Cult. Geogr., 18, 1067-1085, https://doi.org/10.1080/14649365.2016.1210667, 2017.

Piekut, A., Rees, P., Valentine, G., and Kupiszewski, M.: Multidimensional diversity in two European cities: thinking beyond ethnicity, Environ. Plann. A, 44, 2988-3009, https://doi.org/10.1068/a4512, 2012.

Ralph, D. and Staeheli, L. A.: Home and migration: mobilities, belongings and identities, Geography Compass, 5, 517-530, https://doi.org/10.1111/j.1749-8198.2011.00434.x, 2011.
Schiefer, D. and van der Noll, J.: The essentials of social cohesion: a literature review, Soc. Indic. Res., 132, 579-603, https://doi.org/10.1007/s11205-016-1314-5, 2017.

Schlesier, E.: Die Begriffe „Papua“ und „Melanesier“ in den anthropologischen Wissenschaften, Z. Ethnol., 95, 12-24, 1970.

Schneider, J., Crul, M., und Lelie, F.: Generation Mix. Die superdiverse Zukunft unserer Städte - und was wir daraus machen, Waxmann, Münster, New York, 2015.

Simic, A.: The role of superdiverse home country cities in helping migrants negotiate life in superdiverse host country cities, Geoforum, 107, 179-187, https://doi.org/10.1016/j.geoforum.2019.07.015, 2019.

Simpson, E. H.: Measurement of diversity, Nature, 163, p. 688 https://doi.org/10.1038/163688a0, 1949.

Stillwell, J.: Ethnic population concentration and net migration in London, Environ. Plann. A, 42, 1439-1456, https://doi.org/10.1068/a438, 2010.

Vertovec, S.: Super-diversity and its implications, Ethnic Racial Stud., 30, 1024-1054, https://doi.org/10.1080/01419870701599465, 2007.

Vertovec, S.: Talking around super-diversity, Ethnic Racial Stud., 42, 125-139, https://doi.org/10.1080/01419870.2017.1406128, 2019.

White, P.: The settlement patterns of developed world migrants in London, Urban Stud., 35, 1725-1744, https://doi.org/10.1080/0042098984123, 1998.

Wurm, S. A.: Papuan languages of Oceania, Gunter Narr Verlag, Tübingen, 1982. 